\title{
CORRECTIONS
}

\section{A national early warning score for acutely ill patients}

This Editorial (BMJ 2012;345:e5310, doi:10.1136/bmj.e5310) contains an error in the figure "National early warning score and classification of clinical risk." Under the "National early warning score" column, the aggregate for low clinical risk

should have been 1-4 [not 1-3 as was published] 